\title{
Impact of Radii Ratios on a Two-Dimensional Cloaking Structure and Corresponding Analysis for Practical Design at Optical Wavelengths
}

\author{
Nadia Anam and Ebad Zahir \\ American International University-Bangladesh (AIUB), Kemal Ataturk Avenue, Banani, Dhaka 1213, Bangladesh \\ Correspondence should be addressed to Nadia Anam; neela1303@yahoo.com and Ebad Zahir; ebad.zahir@aiub.edu
}

Received 11 September 2016; Accepted 20 December 2016; Published 23 January 2017

Academic Editor: Kin Seng Chiang

Copyright (C) 2017 Nadia Anam and Ebad Zahir. This is an open access article distributed under the Creative Commons Attribution License, which permits unrestricted use, distribution, and reproduction in any medium, provided the original work is properly cited.

\begin{abstract}
This work is an extension to the evaluation and analysis of a two-dimensional cylindrical cloak in the Terahertz or visible range spectrum using Finite Difference Time-Domain (FDTD) method. It was concluded that it is possible to expand the frequency range of a cylindrical cloaking model by careful scaling of the inner and outer radius of the simulation geometry with respect to cell size and/or number of time steps in the simulation grid while maintaining appropriate stability conditions. Analysis in this study is based on a change in the radii ratio, that is, outer radius to inner radius, of the cloaking structure for an array of wavelengths in the visible spectrum. Corresponding outputs show inconsistency in the cloaking pattern with respect to frequency. The inconsistency is further increased as the radii ratio is decreased. The results also help to establish a linear relationship between the transmission coefficient and the real component of refractive index with respect to different radii ratios which may simplify the selection of the material for practical design purposes. Additional performance analysis is carried out such that the dimensions of the cloak are held constant at an average value and the frequency varied to determine how a cloaked object may be perceived by the human eye which considers different wavelengths to be superimposed on each other simultaneously.
\end{abstract}

\section{Introduction}

As recent as a decade ago, the idea of making something invisible seemed fitting to the world of fiction only but some revolutionary work in the field of artificially engineered materials called "metamaterials" is gradually bringing this idea into the real world scenario. Metamaterials consist of periodically or randomly structured subunits whose size and separation are much smaller than the wavelength of an electromagnetic field. Consequently, microscopic details of individual structure elements cannot be sensed by the field, but the average of the assembly's collective response matters. The electromagnetic response of this kind of material can be characterized by an effective relative permittivity and permeability. What makes the metamaterials attractive is the fact that the effective permeability can have nonunity and even negative values at the optical wavelengths. In addition, the effective material parameters can be controlled using properly designed structures [1] and suitable materials [2].
The implications of the practical realization of such a system is vast and thus a matter of great interest to researchers. Practical designs [3-5] have already been made and different methods of analysis used for such designs. An increasingly popular numerical method due to its simplicity yet accurate outputs is the Finite Difference Time-Domain method.

\section{Materials and Methods}

2.1. Metamaterial Cloaking. Cloaking is the ability to make a region of space, and everything in it, invisible to an external observer. A true cloak allows the clear observation of the space behind the cloaked region, and the cloaked region casts no shadow and produces no wavefront changes in the light that has passed through the cloaked region. Cloaking cannot be achieved with materials that exist in nature as they are unable to exhibit negative permittivity and permeability which would lead to a negative index of refraction. Negative 


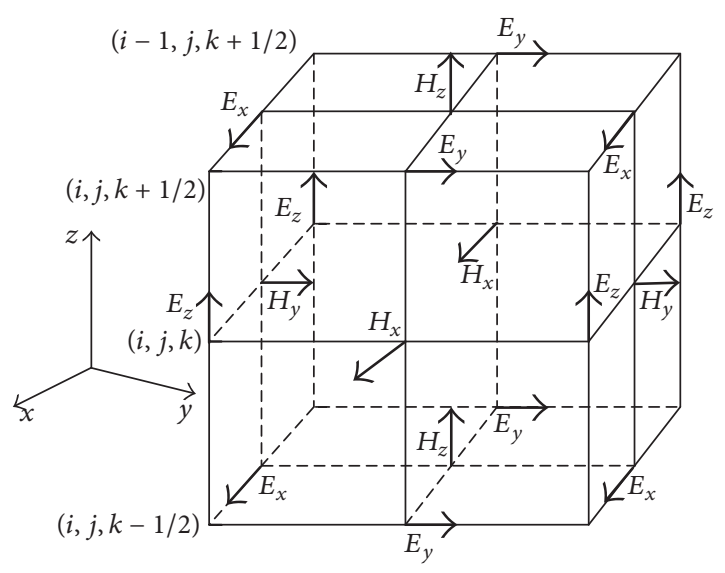

FIGURE 1: Yee's arrangement of field components in a cubic lattice [9].

refractive index is necessary for the way in which light needs to turn around the object that has to be cloaked. A probable solution to this requirement is the use of mematerials. A metamaterial is an artificially structured material which attains its properties from the unit structure rather than the constituent materials. An ordinary material responds to an electric or magnetic field according to the polarization of the atoms and molecules in that material. The structural units of metamaterials can be tailored in shape and size, their composition and morphology can be artificially tuned, and inclusions can be designed and placed in a predetermined manner to achieve prescribed functionalities [3]. In metamaterials, the atoms and molecules are replaced by slightly larger elements which have a physical structure of their own. The response of atoms and molecules is duplicated using tiny circuits [6].

\subsection{Finite Difference Time-Domain Cloaking Using a Cylindri-} cal Structure. The Finite-Difference Time-Domain (FDTD) method achieved discretization of Maxwell's equations in the space and time dimensions. The method is able to solve Maxwell's equations in the time domain for complex structures and geometries [7, 8]. Simulation of electromagnetic waves is made possible by creating a cubic lattice and assigning a staggered arrangement of $E$ and $H$ components to the nodes such as the one shown in Figure 1.

The construction, operation, and characteristics of a $2 \mathrm{D}$ cylindrical cloaking structure have been extensively studied in $[2,10-12]$. For such a cloaking structure in the Transverse Electric (TE) mode, the customized equations for $E$ and $H$ are [12]

$$
\begin{aligned}
E_{x}^{n+1}= & a_{e}\left(D_{x}^{n+1}-2 D_{x}^{n}+D_{x}^{n-1}\right)+b_{e}\left(D_{x}^{n+1}-D_{x}^{n-1}\right) \\
& +c_{e}\left(2 E_{x}^{n}-E_{x}^{n-1}\right)+d_{e}\left(2 E_{x}^{n}+E_{x}^{n-1}\right)+e_{e} E_{x}^{n-1}
\end{aligned}
$$

where

$$
\begin{aligned}
& a_{e}=\frac{4}{g} \\
& b_{e}=\frac{\gamma(2 \Delta t)}{g},
\end{aligned}
$$

$$
\begin{aligned}
c_{e} & =\frac{4 \varepsilon_{0} \varepsilon_{\infty}}{g}, \\
d_{e} & =\frac{\varepsilon_{0} \omega_{p}^{2}(\Delta t)^{2}}{g}, \\
e_{e} & =\frac{\varepsilon_{0} \varepsilon_{\infty} \gamma_{e}(2 \Delta t)}{g}, \\
g & =4 \varepsilon_{0} \varepsilon+\varepsilon_{0} \omega_{p}^{2}(\Delta t)^{2}+\varepsilon_{0} \varepsilon \gamma(2 \Delta t) ;
\end{aligned}
$$

here, $D$ is the electric flux density, $\omega_{p}$ is the plasma frequency, $\gamma$ is the collision frequency, and $\Delta t$ is the size of each time step in the FDTD grid.

Similarly, the magnetic field strength, $H$ can be calculated using

$$
\begin{aligned}
H_{y}^{n+1}= & a_{m}\left(B_{x}^{n+1}-2 B_{y}^{n}+B_{y}^{n-1}\right)+b_{m}\left(B_{y}^{n+1}-B_{y}^{n-1}\right) \\
& +c_{m}\left(2 H_{y}^{n}-H_{y}^{n-1}\right)+d_{m}\left(2 H_{y}^{n}+H_{y}^{n-1}\right) \\
& +e_{m} H_{y}^{n-1} .
\end{aligned}
$$

The FDTD model in $[13,14]$ uses the TM mode for cloaking. The cloaking parameters are therefore described by

$$
\begin{aligned}
& \mu_{r}(r)=\frac{r-r_{a}}{r}, \\
& \mu_{\varphi}(r)=\frac{r}{r-r_{a}}, \\
& \varepsilon_{z}(r)=\left(\frac{r_{b}}{r_{b}-r_{a}}\right)^{2} \frac{r-r_{a}}{r},
\end{aligned}
$$

where $r_{a}$ and $r_{b}$ are the inner and outer radius, respectively, of the cylindrical cloak and $r$ is an arbitrary radius within these two boundary values.

$E_{z}, H_{x}$, and $H_{y}$ can be calculated from corresponding flux densities equations:

$$
\begin{aligned}
& D_{z}^{n+1}[i, j]=D_{z}^{n}[i, j]+\frac{\Delta t}{\Delta}\left(H_{y}^{n+1 / 2}\left[i+\frac{1}{2}, j\right]\right. \\
& \quad-H_{y}^{n+1 / 2}\left[i-\frac{1}{2}, j\right]-H_{x}^{n+1 / 2}\left[i, j+\frac{1}{2}\right] \\
& \left.\quad+H_{x}^{n+1 / 2}\left[i, j-\frac{1}{2}\right]\right), \\
& B_{x}^{n+1 / 2}\left[i, j+\frac{1}{2}\right]=B_{x}^{n-1 / 2}\left[i, j+\frac{1}{2}\right] \\
& \quad+\frac{\Delta t}{\Delta}\left(-E_{z}^{n}[i, j+1]+E_{z}^{n}[i, j]\right) \\
& B_{y}^{n+1 / 2}\left[i+\frac{1}{2}, j\right]=B_{x}^{n-1 / 2}\left[i+\frac{1}{2}, j\right]+\frac{\Delta t}{\Delta}\left(E_{z}^{n}[i+1, j]\right. \\
& \left.\quad-E_{z}^{n}[i, j]\right) .
\end{aligned}
$$

The index of refraction was calculated from

$$
n_{\mathrm{FDTD}}=\frac{1}{j k_{0}\left(z_{1}-z_{2}\right)} \log \left|\frac{E_{x}\left(\omega, z_{2}\right)}{E_{x}\left(\omega, z_{1}\right)}\right|,
$$


TABLE 1: Parametric variations considered for the study of the FDTD cloaking model.

\begin{tabular}{lcccccc}
\hline$r_{a}$ and $r_{b}$ & Frequency & $\Delta$ & $\Delta t$ & $I$ and $J$ & $S_{c}$ & PML \\
\hline Varied & Fixed & Fixed & Fixed & Fixed & Fixed & Fixed \\
Fixed & Fixed & Fixed & Varied & Fixed & Varied & Fixed \\
Fixed & Varied & Varied & Varied & Fixed & Fixed & Fixed \\
Varied & Varied & Varied & Varied & Fixed & Fixed & Fixed \\
Varied & Varied & Varied & Varied & Varied & Fixed & Fixed \\
\hline
\end{tabular}

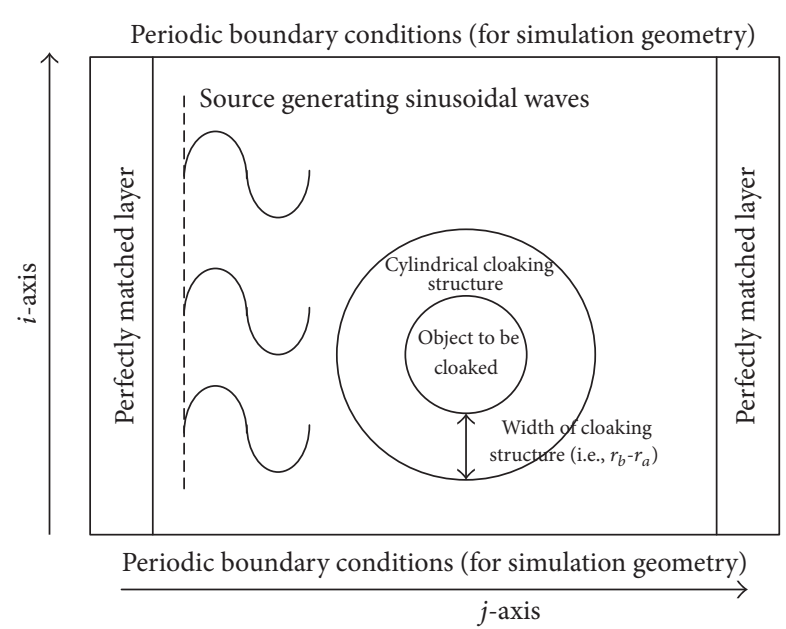

FIGURE 2: Simulation geometry for FDTD method of cloaking [10, 11].

where $\omega$ is the angular frequency, $k_{0}$ is the wave number set as $\omega_{0} / c\left(\omega_{0}\right.$ is the angular frequency of the sinusoidal source wave), and the fields were recorded at locations $z_{1}=1415 \Delta z$ and $z_{2}=1424 \Delta z$ ( $\Delta z$ is the spatial step).

In order to expand the range of the FDTD cloaking model from a few Gigahertz to hundreds of Terahertz, it was essential to understand the scope of the simulation parameters. The inner and outer radii of the structure $r_{a}$ and $r_{b}$, cell size in the $x$ and $y$ directions $\Delta$, the temporal steps $\Delta t$, the spatial steps in the $i-j$ axis $I$ and $J$, the courant stability $S_{c}$, and the perfectly matched layer width PML were some of the prime parameters taken into consideration and several test analyses were performed. A summary is presented in Table 1 and the simulation geometry is shown in Figure 2. Due to reflection restraints from the boundaries, the PML layer(s) needed to be kept constant.

The equation that governs the stability of the FDTD system is

$$
S_{c}=u \frac{\Delta t}{\Delta}
$$

where $u$ is the speed of light in any medium and is taken to be equal to $c\left(=2.998 \times 10^{8} \mathrm{~m} / \mathrm{s}^{2}\right)$ since light is considered to be propagating through air medium. Altering the stability suggests that the closest to ideal cloaking can be achieved if the stability value is the lowest possible.
However, maintaining a very low value would make the design excessively stringent due to extremely small time step requirements for high frequency values. At the same time, cloaking tends to become more unreliable with higher stability number so it is proposed that the number be not allowed to exceed 0.5 [15]. For this study it is considered to be 0.25 . The ratio of the outer radius to inner radius $\left(r_{b} / r_{a}\right)$ has been varied in the same ratio as the cell size and/or the number of time steps taking into account that the structural units of the metamaterial must be substantially smaller than the wavelength being considered [3]. The cell size $\Delta$ can be related to the wavelength and hence frequency by

$$
\Delta=\frac{\lambda}{50} .
$$

The equation is formed by implying the relevant relationships and test conditions suggested in [10,12] and modifying it to match the default frequency and cell size values used in $[13,14]$. Thus at optical frequencies, the cell size is reduced and the time steps increased accordingly to hold the stability constant at the preconsidered value. Using (8), the stability relation in (7) may be redefined as

$$
S_{c}=50 \times f \times \Delta t,
$$

where $f$ is the frequency of light waves.

It is also suggested in [12] that the ratio of $r_{b} / r_{a}$ should be maintained at 2 to obtain reliable cloaking results. To reduce design costs, however, it would be beneficial to set the ratio as close to 1 as possible. The first part of the study here incorporates this idea; three different radii ratios 1.5 , 1.75 and 2 are considered for different optical wavelengths in the visible spectrum to observe the level of cloaking for specific colors of light and whether the radii ratio has any impact on the process. For compatibility of the design with our visual capability where the human eye perceives different colors of light simultaneously, the size and hence dimensions of the cloaked object should be constant and not varied with wavelength. This leads to the latter part of the research, where the inner and outer radius of the cloak are fixed and the frequency varied over the entire visible spectrum. To preserve simulation geometry constraints, however, the cell size still needs to be varied with frequency and this in turn requires the number of time steps to be varied so that stability is maintained at the desired value.

The plots in Figures 3-5 depict the changes in the transmitted sinusoidal waveform when no object is present in its path and it has a frequency of $2 \mathrm{GHz}$, when a cylindrical cloak of $r_{a}=0.1 \mathrm{~m}$ and $r_{b}=0.2 \mathrm{~m}$ is situated in the centre of the simulation geometry with the wave frequency still $2 \mathrm{GHz}$ and when the propagating wave has a frequency of $545 \mathrm{THz}$ with $r_{a}=0.367 \mu \mathrm{m}$ and $r_{b}=0.733 \mu \mathrm{m}$. It is worth noting in Figure 5 that, due to the increase in frequency, the $j$-axis limits have been doubled for complete observation of the propagating wave from crest to trough. The cloak is also considered to be "lossless" suggesting that there is no degradation in the intensity of the reflected light compared to the incident one. 


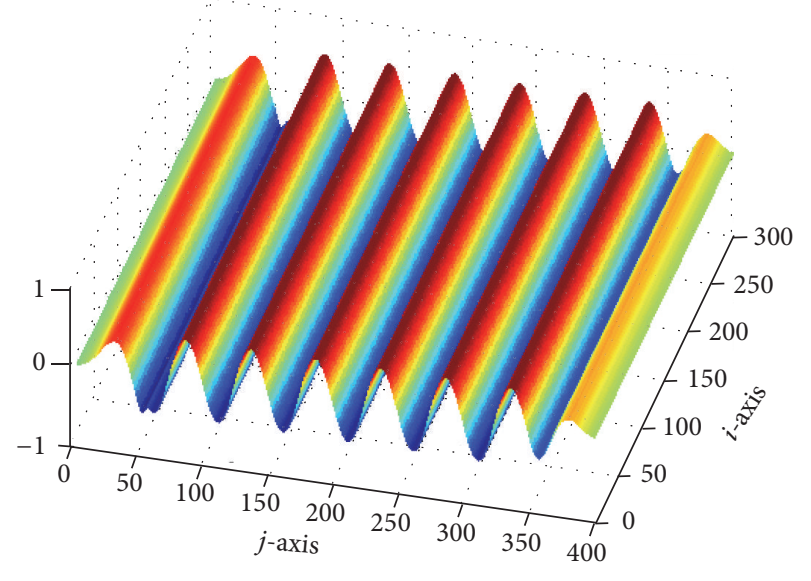

FIGURE 3: Three-dimensional field distribution plot for a $2 \mathrm{GHz}$ sine wave with no object in its path.

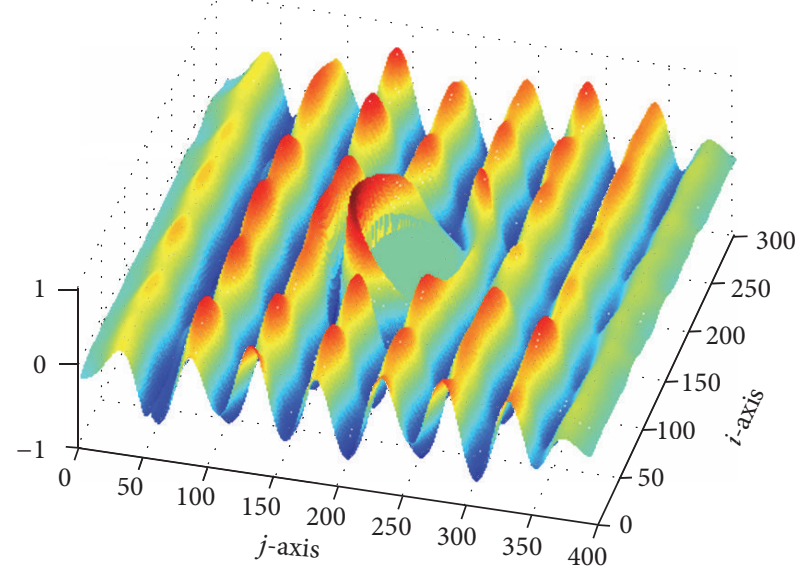

FIgURE 4: Three-dimensional field distribution plot for a $2 \mathrm{GHz}$ sine wave with a steady lossless cylindrical cloak in its path.

\section{Results and Discussion}

The model in [13] provides the waveforms of transmitted field, transmitted field beyond the cylindrical slab, that is, the cloaked object, transmission coefficient, reflection coefficient (calculated by subtracting transmission coefficient from unity), and the real and imaginary components of the refractive index. The transmitted fields are determined by averaging the waveforms at various time steps. The transmission and reflection coefficients and refractive indices are calculated at specific optical wavelengths or colors of light as shown in the tables. Tables 2-4 are constructed using three different radii ratios $\left(r_{b} / r_{a}\right) 2,1.75$, and 1.5 , respectively. Outputs at a ratio lower than 1.5 are fairly unreliable so they are excluded from this study. Table 5 uses a fixed radius ratio of 2 as well as fixed average radius values $\left(r_{a}=0.365 \mu \mathrm{m}\right.$ and $\left.r_{b}=0.730 \mu \mathrm{m}\right)$ for various wavelengths.

The outcomes of Tables 2-4 are summarized in Figures 610. Figure 6 displays the difference between the transmitted field prior to its entrance into the cylindrical slab and that beyond it with respect to frequency. For ideal cloaking, this

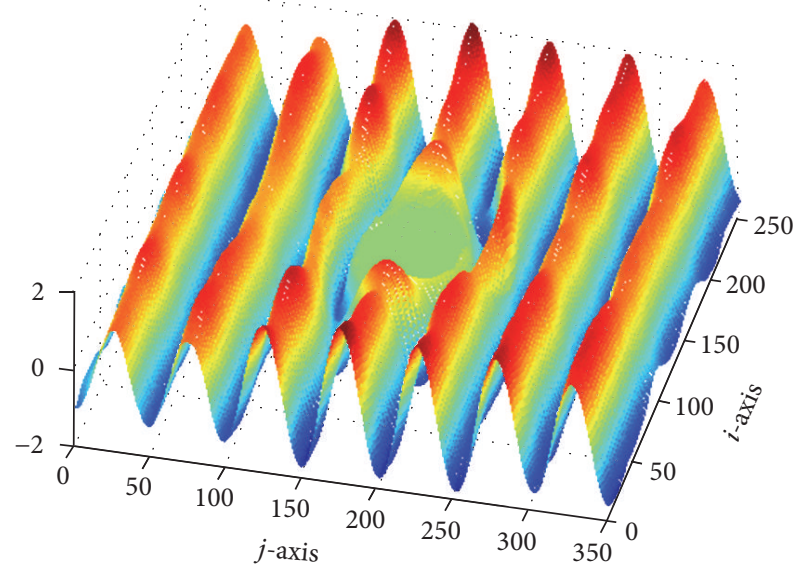

FIgURE 5: Three-dimensional field distribution plot for a $545 \mathrm{THz}$ sine wave with a steady lossless cylindrical cloak in its path and appropriately scaled parameters.

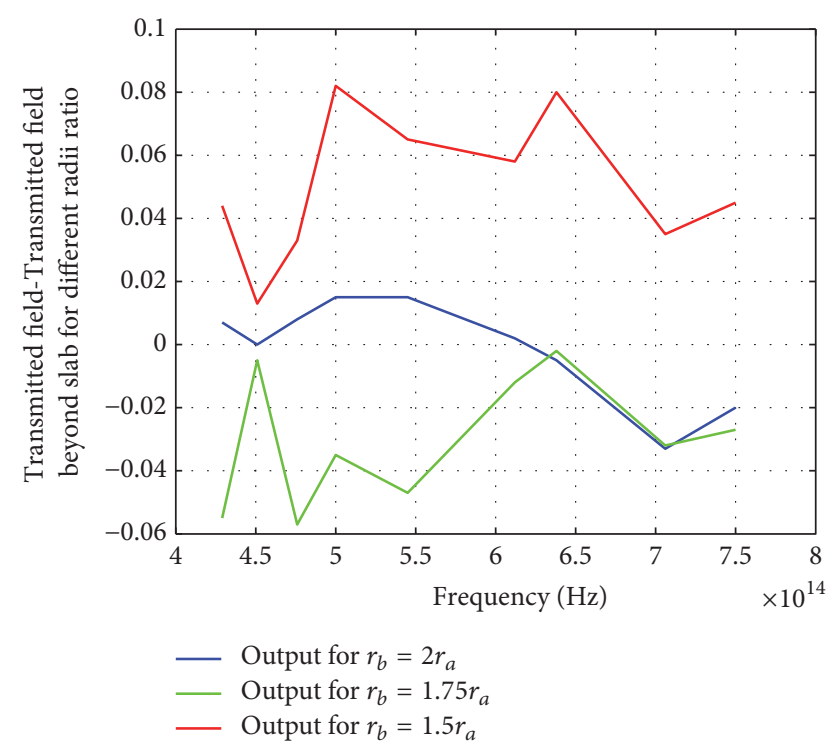

FIGURE 6: Plots for transmitted field-transmitted field beyond slab versus frequency for different radii ratio.

value should be as close to 0 as possible. For all three radii ratio, red color is cloaked most accurately, with the highest accuracy for $r_{b}=2 r_{a}$. This high accuracy is obtained again at a wavelength near to aqua but only for a radius ratio of 2. At this same frequency, the accuracy deviates almost 1 percent for $r_{b}=1.75 r_{a}$ and almost 6 percent for $r_{b}=$ $1.5 r_{a}$. For $r_{b}=2 r_{a}$, the maximum deviation occurs around indigo light (3.5 percent approximately); for $r_{b}=1.75 r_{a}$, maximum inaccuracy is obtained at orange light (5.8 percent approximately) and for $r_{b}=1.5 r_{a}$; this point occurs at yellow light (8.1 percent approximately).

Figure 7 plots transmission coefficients with respect to different optical frequencies. The ideal value is 1 and the behavioral pattern obtained in Figure 6 follows with a few discrepancies, significant of which is the sharp plunge in value to almost 0.68 for blue light at $r_{b}=1.5 r_{a}$. One reason may 
TABLE 2: Variations in different parameters of an optical cloaking system for a radius ratio of 2.

\begin{tabular}{lcccccccc}
\hline Color of light & $\begin{array}{c}\text { Wavelength } \\
(\mathrm{nm})\end{array}$ & $\begin{array}{c}\text { Frequency } \\
\text { (THz) }\end{array}$ & $\begin{array}{c}\text { Transmitted } \\
\text { field }\end{array}$ & $\begin{array}{c}\text { Transmitted } \\
\text { field beyond } \\
\text { slab }\end{array}$ & $\begin{array}{c}\text { Transmission } \\
\text { coefficient }\end{array}$ & $\begin{array}{c}\text { Reflection } \\
\text { coefficient }\end{array}$ & $\begin{array}{c}\text { Refractive } \\
\text { index (real) }\end{array}$ & $\begin{array}{c}\text { Refractive } \\
\text { index } \\
\text { (imaginary) }\end{array}$ \\
\hline Dark red & 700 & 429 & 0.712 & 0.705 & 1.04 & -0.04 & 1.9 & 0.5 \\
Red & 665 & 451 & 0.698 & 0.698 & 1 & 0 & 1.8 & 0.5 \\
Orange & 630 & 476 & 0.703 & 0.695 & 1.07 & -0.07 & 1.95 & 0.56 \\
Yellow & 600 & 500 & 0.713 & 0.698 & 1.02 & -0.02 & 1.85 \\
Green & 550 & 545 & 0.73 & 0.715 & 1.08 & -0.08 & 1.97 & 0.5 \\
Aqua & 490 & 612 & 0.705 & 0.703 & 1 & 0 & 0.58 \\
Blue & 470 & 638 & 0.723 & 0.728 & 1.04 & -0.04 & 1.8 & 0.45 \\
Indigo & 425 & 706 & 0.675 & 0.708 & 1.06 & -0.06 & 1.95 \\
Violet & 400 & 750 & 0.72 & 0.74 & 1.03 & -0.03 & 0.51 \\
\hline
\end{tabular}

TABLE 3: Variations in different parameters of an optical cloaking system for a radius ratio of 1.75.

\begin{tabular}{lcccccccc}
\hline Color of light & $\begin{array}{c}\text { Wavelength } \\
(\mathrm{nm})\end{array}$ & $\begin{array}{c}\text { Frequency } \\
(\mathrm{THz})\end{array}$ & $\begin{array}{c}\text { Transmitted } \\
\text { field }\end{array}$ & $\begin{array}{c}\text { Transmitted } \\
\text { field beyond } \\
\text { slab }\end{array}$ & $\begin{array}{c}\text { Transmission } \\
\text { coefficient }\end{array}$ & $\begin{array}{c}\text { Reflection } \\
\text { coefficient }\end{array}$ & $\begin{array}{c}\text { Refractive } \\
\text { index (real) }\end{array}$ & $\begin{array}{c}\text { Refractive } \\
\text { index } \\
\text { (imaginary) }\end{array}$ \\
\hline Dark red & 700 & 429 & 0.725 & 0.78 & 1.1 & -0.1 & 2.55 \\
Red & 665 & 451 & 0.758 & 0.763 & 1.05 & -0.05 & 2.5 & 0.36 \\
Orange & 630 & 476 & 0.728 & 0.785 & 1.15 & -0.15 & 2.7 & 0.4 \\
Yellow & 600 & 500 & 0.735 & 0.77 & 1.05 & -0.05 & 2.5 & 0.38 \\
Green & 550 & 545 & 0.743 & 0.79 & 1.13 & -0.13 & 2.65 & 0.38 \\
Aqua & 490 & 612 & 0.738 & 0.75 & 1.01 & -0.01 & 2.4 & 0.38 \\
Blue & 470 & 638 & 0.743 & 0.745 & 1.1 & -0.1 & 2.6 & 0.39 \\
Indigo & 425 & 706 & 0.733 & 0.765 & 1.14 & -0.14 & 2.64 & 0.4 \\
Violet & 400 & 750 & 0.713 & 0.74 & 1.05 & -0.05 & 2.5 \\
\hline
\end{tabular}

TABLE 4: Variations in different parameters of an optical cloaking system for a radius ratio of 1.5.

\begin{tabular}{lcccccccc}
\hline Color of light & $\begin{array}{c}\text { Wavelength } \\
(\mathrm{nm})\end{array}$ & $\begin{array}{c}\text { Frequency } \\
(\mathrm{THz})\end{array}$ & $\begin{array}{c}\text { Transmitted } \\
\text { field }\end{array}$ & $\begin{array}{c}\text { Transmitted } \\
\text { field beyond } \\
\text { slab }\end{array}$ & $\begin{array}{c}\text { Transmission } \\
\text { coefficient }\end{array}$ & $\begin{array}{c}\text { Reflection } \\
\text { coefficient }\end{array}$ & $\begin{array}{c}\text { Refractive } \\
\text { index (real) }\end{array}$ & $\begin{array}{c}\text { Refractive } \\
\text { index } \\
\text { (imaginary) }\end{array}$ \\
\hline Dark red & 700 & 429 & 0.762 & 0.718 & 1.11 & -0.11 & 3.14 & 0.08 \\
Red & 665 & 451 & 0.753 & 0.74 & 1.04 & -0.05 & 3.1 & 0.1 \\
Orange & 630 & 476 & 0.753 & 0.72 & 1.15 & -0.15 & 3.15 & 0 \\
Yellow & 600 & 500 & 0.765 & 0.683 & 1.08 & -0.08 & 3.14 & 0.03 \\
Green & 550 & 545 & 0.778 & 0.713 & 1.15 & -0.15 & 3.2 & 0 \\
Aqua & 490 & 612 & 0.753 & 0.695 & 1.04 & -0.04 & 3.1 & 0.13 \\
Blue & 470 & 638 & 0.78 & 0.7 & 0.67 & 0.33 & 2.1 & 0.58 \\
Indigo & 425 & 706 & 0.755 & 0.72 & 1.14 & -0.14 & 3.15 & 0 \\
Violet & 400 & 750 & 0.783 & 0.738 & 1.13 & -0.13 & 3 \\
\hline
\end{tabular}

be the dependency of the metamaterial structure and hence its response on the operational frequency [16]. The reduction in the thickness of the metamaterial cloak impacts its overall response and causes incongruous behavior at blue light. The best results overall remain the same as those depicted by Figure 6; that is, $r_{b}=2 r_{a}$ shows minimum deviations.
Figure 8 shows reflection coefficient values that have been directly calculated from the relationship $T=1-R$ where $T=$ transmission coefficient and $R=$ reflection coefficient.

The plots in Figures 9 and 10 for refractive index help to determine which materials or metamaterial structures would be suitable for the practical design; the refractive 
TABLE 5: Variations in different parameters of an optical cloaking system for a radius ratio of 2 with fixed inner $\left(r_{a}=0.365 \mu \mathrm{m}\right)$ and outer $\left(r_{b}=0.730 \mu \mathrm{m}\right)$ radius.

\begin{tabular}{lcccccccc}
\hline Color of light & $\begin{array}{c}\text { Wavelength } \\
(\mathrm{nm})\end{array}$ & $\begin{array}{c}\text { Frequency } \\
(\mathrm{THz})\end{array}$ & $\begin{array}{c}\text { Transmitted } \\
\text { field }\end{array}$ & $\begin{array}{c}\text { Transmitted } \\
\text { field beyond } \\
\text { slab }\end{array}$ & $\begin{array}{c}\text { Transmission } \\
\text { coefficient }\end{array}$ & $\begin{array}{c}\text { Reflection } \\
\text { coefficient }\end{array}$ & $\begin{array}{c}\text { Refractive } \\
\text { index (real) }\end{array}$ & $\begin{array}{c}\text { Refractive } \\
\text { index } \\
\text { (imaginary) }\end{array}$ \\
\hline Dark red & 700 & 429 & 0.72 & 0.77 & 1.07 & -0.07 & 1.87 & -0.25 \\
Red & 665 & 451 & 0.738 & 0.784 & 1.04 & -0.04 & 2.13 & -0.33 \\
Orange & 630 & 476 & 0.736 & 0.778 & 1.04 & -0.04 & 2.33 & -0.18 \\
Yellow & 600 & 500 & 0.724 & 0.744 & 1.01 & -0.01 & 2.42 & 0.12 \\
Green & 550 & 545 & 0.688 & 0.71 & 1.02 & -0.02 & 1.9 & 0.48 \\
Aqua & 490 & 612 & 0.772 & 0.826 & 1.1 & -0.1 & 1.37 & -0.13 \\
Blue & 470 & 638 & 0.77 & 0.836 & 1.07 & -0.07 & 1.38 & -0.41 \\
Indigo & 425 & 706 & 0.782 & 0.86 & 1.07 & -0.07 & 1.46 & 1.87 \\
Violet & 400 & 750 & 0.8 & 0.69 & 1.1 & -0.1 & 0 \\
\hline
\end{tabular}

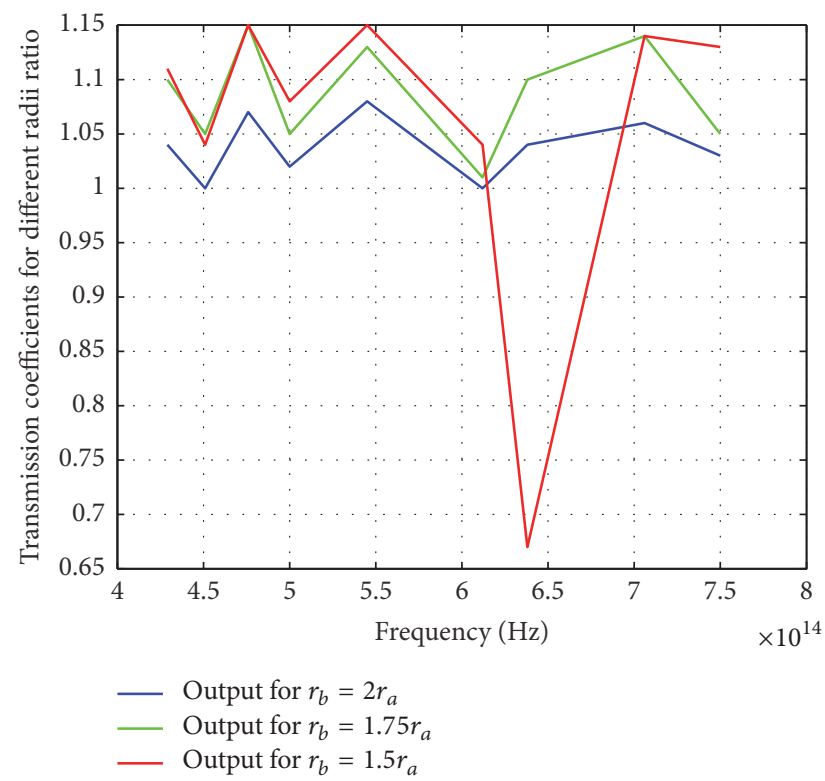

Figure 7: Plots for transmission coefficient versus frequency for different radii ratio.

index is a complex value (with real and imaginary components) as the structure requires negative values of permittivity and permeability at certain angles of reflection for cloaking. As expected, the refractive indices for each radius ratio are different because light's path of curvature would vary for individual cloak size. These also suggest that the practical design of an optical cloak for a radius ratio of either 2 or 1.75 would be simpler compared to that for 1.5 .

The deviations in refractive index with respect to frequency are lower so easier to incorporate into the metamaterial structure. With a radius ratio of 1.5 however, the necessity to overcome the anomaly existing from aqua to indigo light would make the design quite complex.

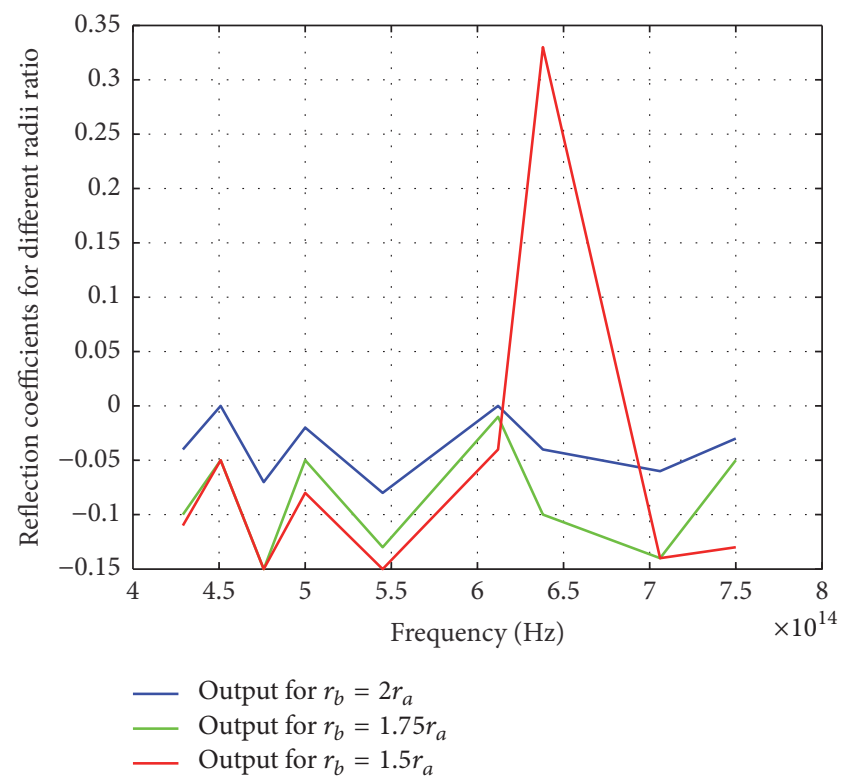

FIGURE 8: Plots for reflection coefficient versus frequency for different radii ratio.

Figures 7 and 9 illustrate an additional important aspect, an almost linear relationship between transmission coefficient and real part of refractive index; a clearer presentation of this pattern is demonstrated in Figures 11-13.

This behavior can be mathematically approximated as

$$
\begin{aligned}
T \cong 1.8 \operatorname{Re}_{\text {real }} & (\text { for a radius ratio of } 2), \\
T \cong 2.3 \operatorname{Re}_{\text {real }} & (\text { for a radius ratio of } 1.75) \\
T \cong 2.8 \operatorname{Re}_{\text {real }} & (\text { for a radius ratio of } 1.5) .
\end{aligned}
$$

Otherwise, it is correlated as

$$
\frac{T_{1}}{\mathrm{Re}_{1}} \times \frac{r_{b 1}}{r_{a 1}} \times k_{1}=\frac{T_{2}}{\mathrm{Re}_{2}} \times \frac{r_{b 2}}{r_{a 2}},
$$




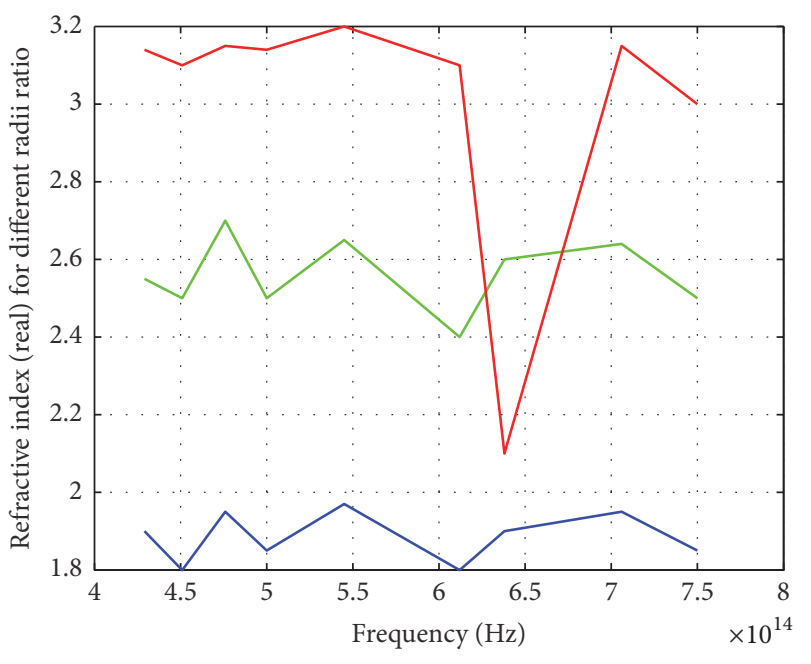

$\begin{aligned} \text { Output for } r_{b} & =2 r_{a} \\ \text { Output for } r_{b} & =1.75 r_{a} \\ \text { Output for } r_{b} & =1.5 r_{a}\end{aligned}$

FIGURE 9: Plots for the real component of refractive index versus frequency for different radii ratio.

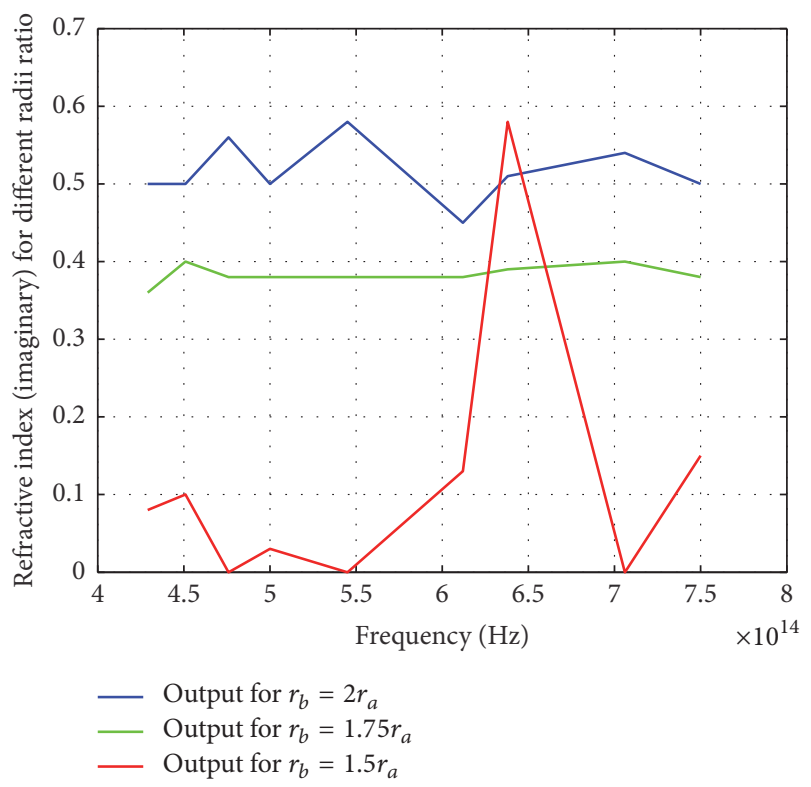

FIgURE 10: Plots for the imaginary component of refractive index versus frequency for different radii ratio.

or

$$
\frac{T_{2}}{\mathrm{Re}_{2}} \times \frac{r_{b 2}}{r_{a 2}} \times k_{2}=\frac{T_{3}}{\mathrm{Re}_{3}} \times \frac{r_{b 3}}{r_{a 3}},
$$

where $k_{1}$ and $k_{2}$ are constants of proportionality. The development of such equations could simplify the choice of the metamaterial structure and composition for the actual design since only the awareness of the radius ratio would be required (and transmission coefficient can be considered to be the ideal value of 1 ).

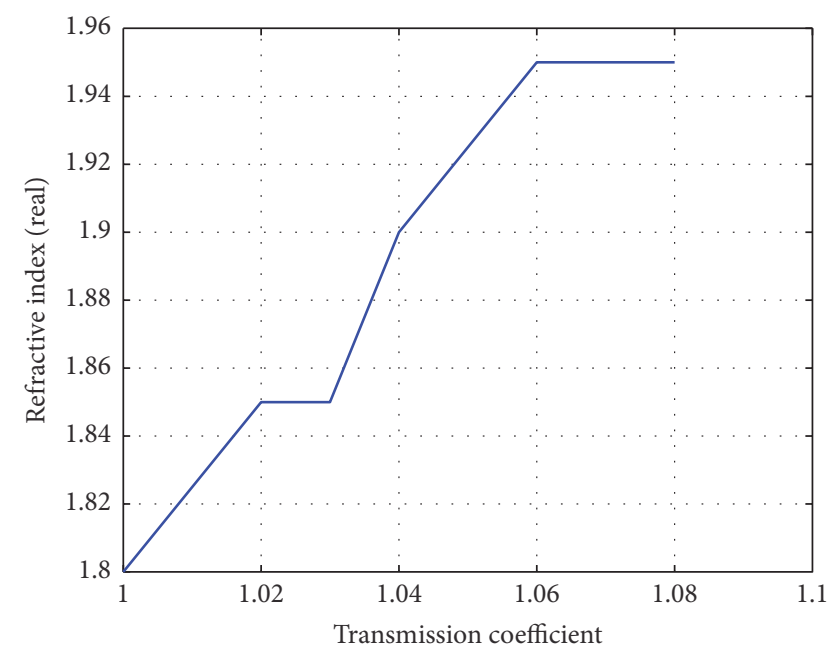

FIGURE 11: Plot depicting an almost linear relationship between the real component of refractive index and transmission coefficient for a radius ratio of 2 .

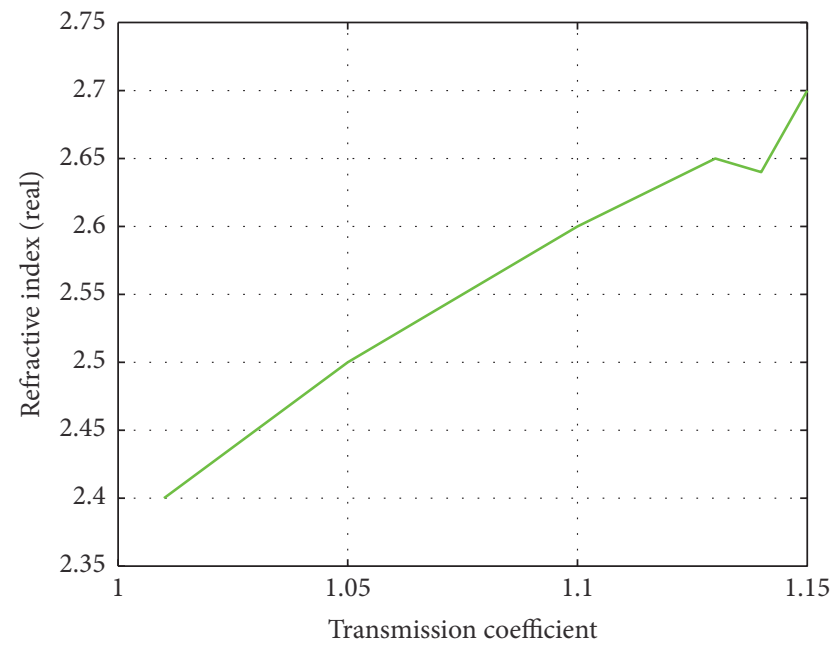

FIGURE 12: Plot depicting an almost linear relationship between the real component of refractive index and transmission coefficient for a radius ratio of 1.75

If an object has to be completely invisible to the human eye, that is, perfect cloaked, the cloaking structure must not only allow the light to pass through it undisturbed but also ensure that it reflects back to the human eye coherently from what object is present beyond this setup. The incident field, transmission field, transmission field beyond slab, transmission and reflection coefficients, and refractive index versus frequency for red, blue, and green light are plotted simultaneously (Figures 14-18) to obtain an understanding of this matter. The radius needs to be held constant for practical purposes and is chosen as an averaged value of those considered in the preceding part of this study for the entire visible spectrum. The output waveforms for the three basic colors appear quite coherent, particularly for the refractive index values, suggesting easier material selection for designing purposes with an expectation of consistent 


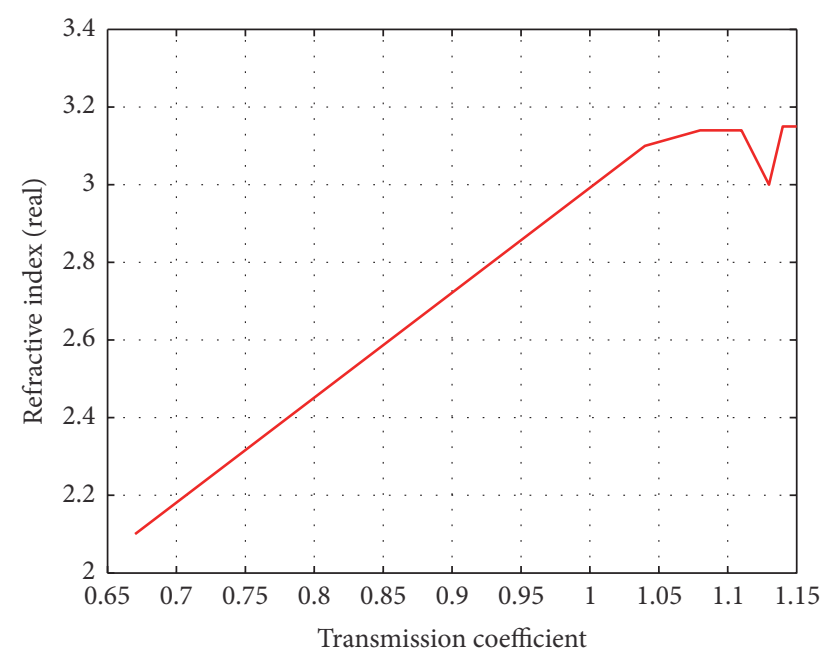

FIGURE 13: Plot depicting an almost linear relationship between the real component of refractive index and transmission coefficient for a radius ratio of 1.5 .
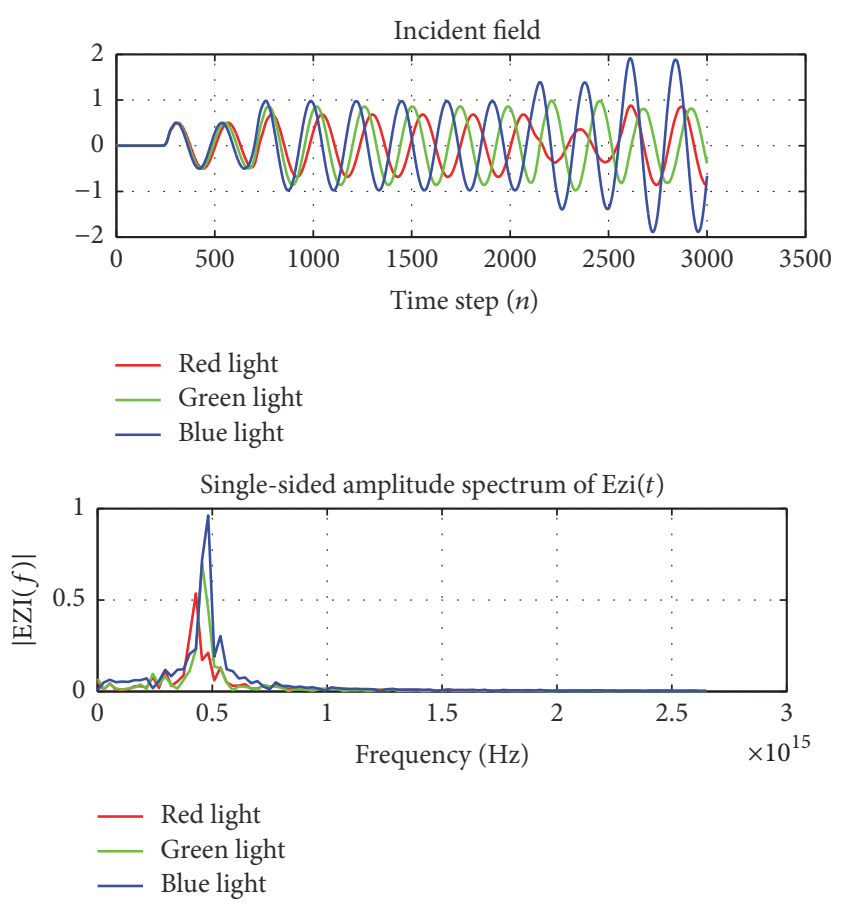

FIGURE 14: Incident field and equivalent amplitude spectrum versus frequency for red, green, and blue light corresponding to conditions in Table 5.

superposition of all wavelengths. The additional frequency spectrum plots in Figures 14-16 also infer that the structure remains fairly "lossless" and hence the modifications that have been made to the model for optical frequency operations are satisfactory.

Plotting further wavelengths of light on these same axes would make it extremely difficult to differentiate each output. Instead, Table 5 and corresponding Figures 19-21 summarize the overall findings.
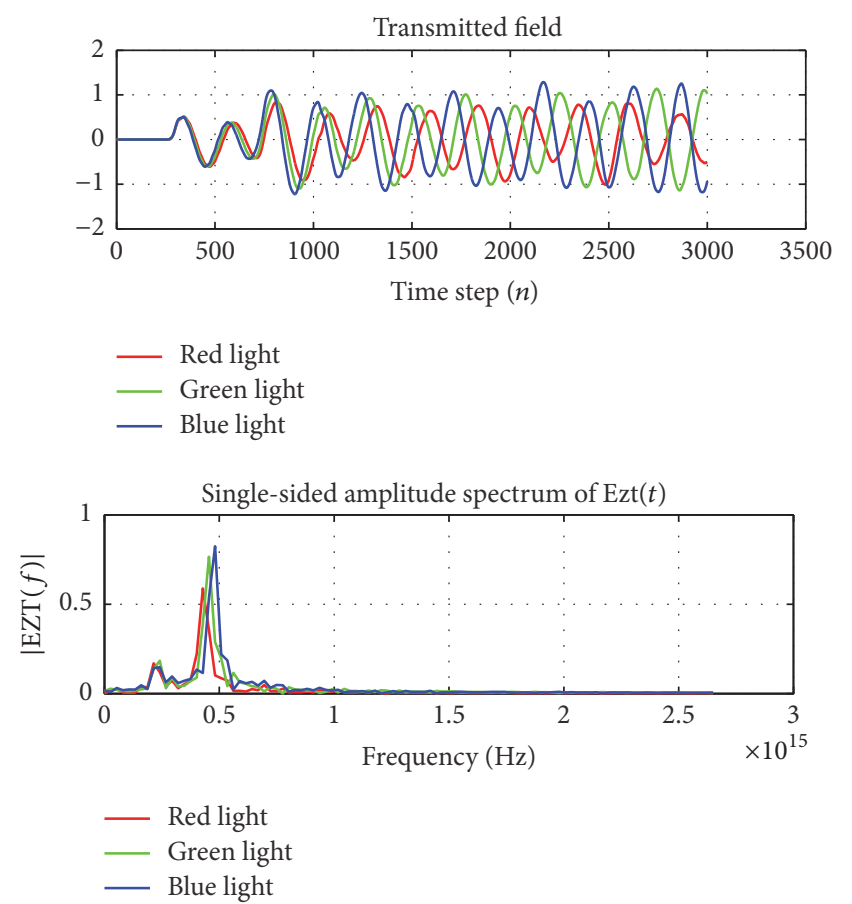

FIGURE 15: Field transmitted into the cloaking slab and equivalent amplitude spectrum versus frequency for red, green, and blue light corresponding to conditions in Table 5.

Table 5 and Figure 10 suggest that while it may be possible to observe almost every color of light in the visible spectrum with minor inaccuracy ( 2 to 7 percent) simultaneously, the overall cloaking quality begins to deteriorate from aqua onwards (inaccuracies rising to 10 percent at maximum). Plots in Figures 12 and 13 present another practical design hurdle; since the real part of refractive index is zero and the imaginary part unobtainable for violet light (shown with arrows), the actual cloaking structure will not be able to reflect violet and other optical wavelengths beyond it unless the radii values are altered.

Due to the frequency independent nature of the coordinate transformation functions used in the FDTD model, the range of the operating frequency can be easily expanded from Gigahertz [12] to Terahertz [10] ranges. Also, altering shapes [17] is quite easily achievable. Such factors are influencing the development of numerous cloaking models based on the FDTD method. However, the performance of such structures can be severely affected if they are not operated at the designed frequency [18]. An ideal cylindrical cloak such as the one in [11] has been proved to work properly for monochromatic incident wave, but when excited with nonmonochromatic radiation, they become nonpractical. Also, losses tend to become significant under such circumstances. The findings in this study are consistent with previous researches and show how the performance of the FDTD model is affected if cloaking parameters are not simultaneously altered with frequencies changes. Since losses are incurred, the object will not be "perfectly" cloaked and 

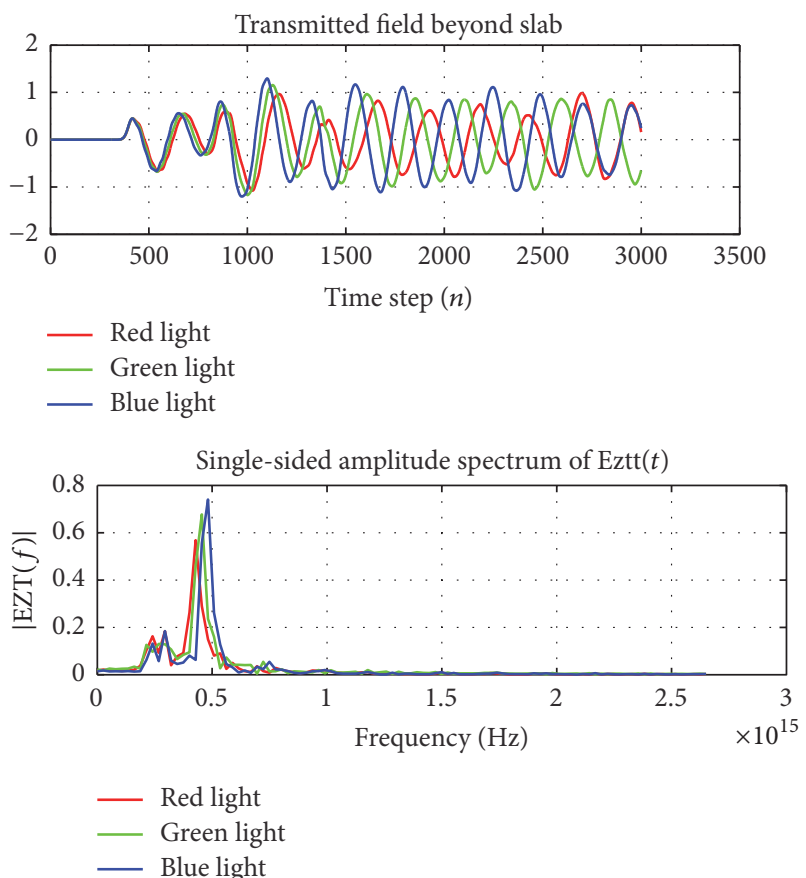

FIgURE 16: Transmitted field beyond the cloaking slab and equivalent amplitude spectrum versus frequency for red, green, and blue light corresponding to conditions in Table 5.

there will be a shadowing effect; that is, the cloak will appear somewhat gray instead of being fully transparent $[11,19]$.

\section{Conclusion}

A previous study of a two-dimensional cylindrical cloak model in the optical frequency or visible spectrum region has been extended here for different radii ratios. The model uses a Finite Difference Time-Domain Method of cloaking and the behavioral transformation for different inner to outer radii ratios in parameters such as transmitted field before and beyond the cloak, transmission and reflection coefficients, and refractive index with respect to different optical wavelengths is studied. Outcomes suggest that the "cloaking" quality is influenced by not just wavelength values but also the radii ratios. If accuracy is most necessary, it is best to use a radius ratio of 2 . However, if design costs and thickness are significant, the ratio can be lowered to 1.75 with minor deterioration in output quality. The results also portray an almost linear relationship between the transmission coefficient and the real component of refractive index with respect to radii ratios, implying that the type of material required for cloaking a specific wavelength of light may be easily found if only the thickness of the cloaking structure is known.

In further investigations, the size of the cloak is kept constant and the wavelength varied to obtain an idea of how light would reflect from an entity beyond the cloaked object and appear to our eyes. Results suggest incoherency in cloaking with an acceptable inaccuracy of around 2 to 10 percent. Moreover, the averaging of output values for three different wavelengths (red, blue, and green) of light
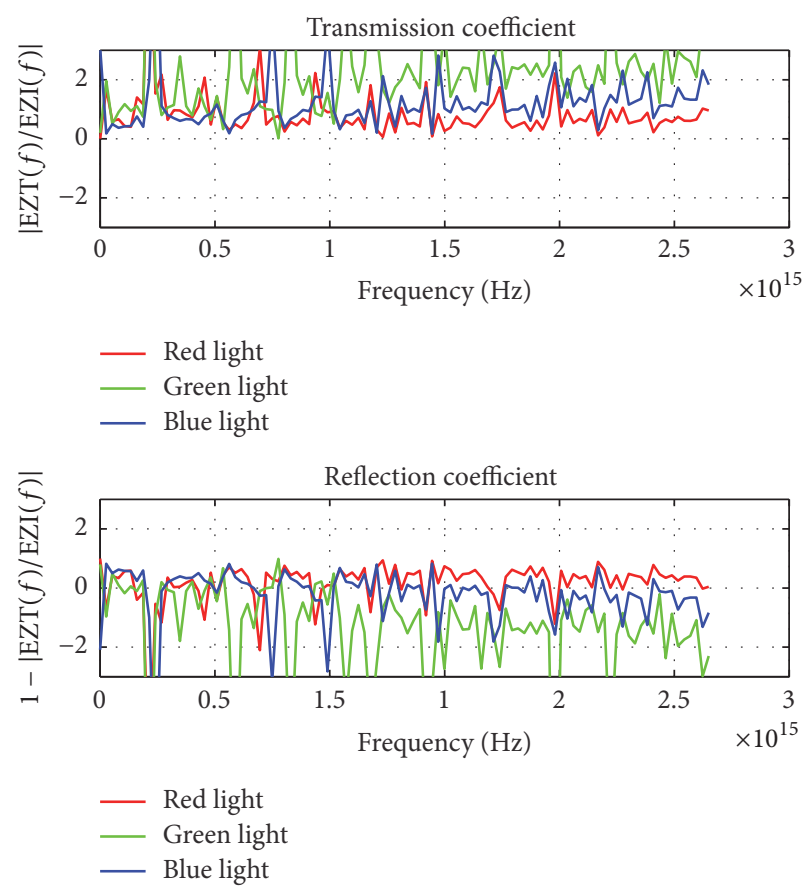

FIGURE 17: Transmission and reflection coefficient versus frequency for red, green, and blue light corresponding to conditions in Table 5.
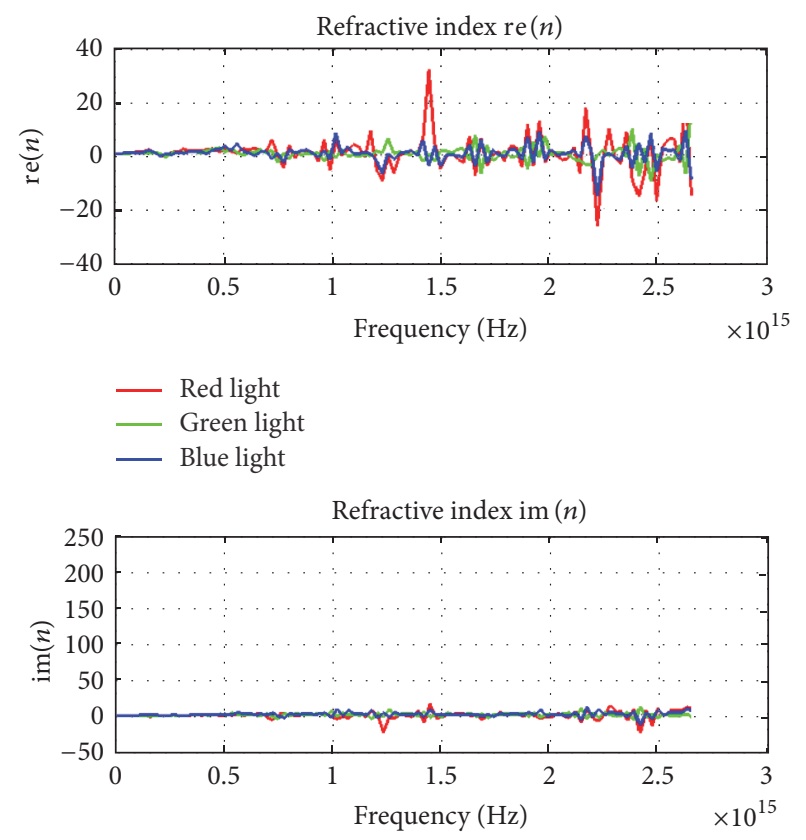

FIGURE 18: Refractive index (real and imaginary) versus frequency for red, green, and blue light corresponding to conditions in Table 5.

suggests that these results may be considered acceptable for the real-life scenario where different wavelengths in the visible spectrum exist in a superimposed form. An important issue, however, is that no outputs can be achieved for violet light and beyond. Thus an entity beyond the cloaked object will not be able to reflect violet light with the average radius 


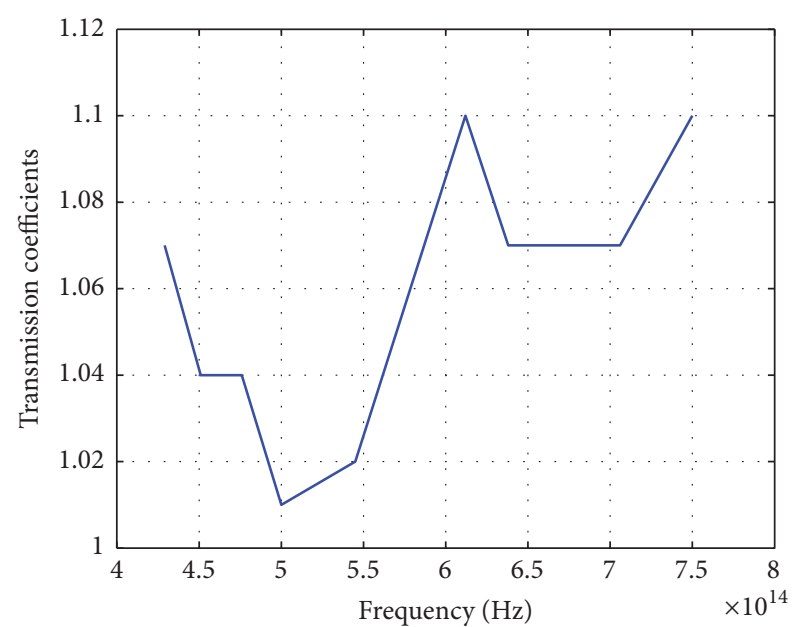

FIGURE 19: Variations in transmission coefficient with frequency for a radius ratio of 2 with fixed inner and outer radius values.

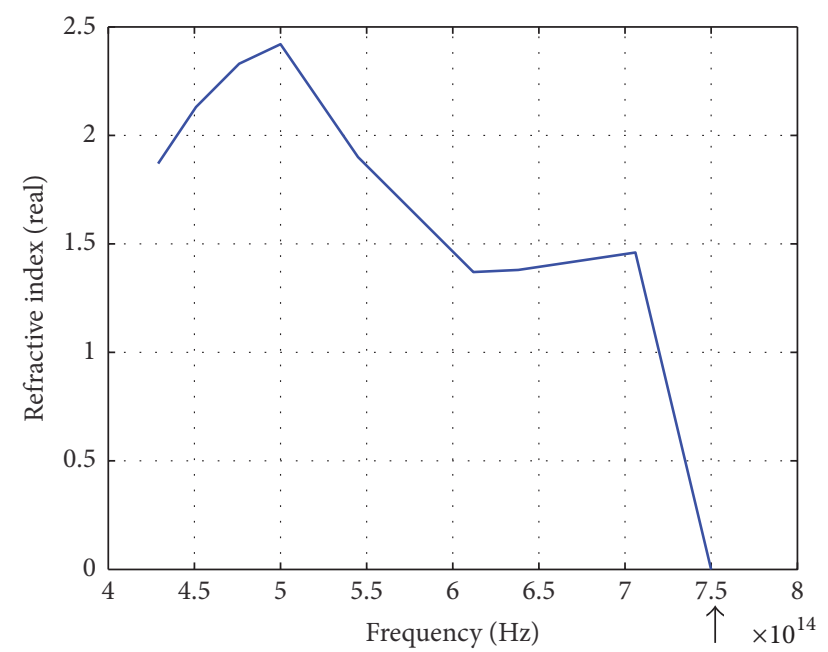

Figure 20: Plot showing how the refractive index (real) changes with frequency for a radius ratio of 2 and fixed inner and outer radius value (arrow shows the value reaches zero at a frequency of $7.5 \mathrm{THz}$ ).

value used here. Also, the size of this cloak is in the micronano range; for this to increase to a considerable dimension, the parameters, boundary conditions, and mathematical equations that govern this two-dimensional model must be altered in such a way that the output is still stable and reliable while the frequency is sustained in the optical range. These restraints could be overcome if the cloak is constructed from suitable "active" metamaterials.

\section{Competing Interests}

The authors declare that there is no conflict of interests regarding the publication of this paper.

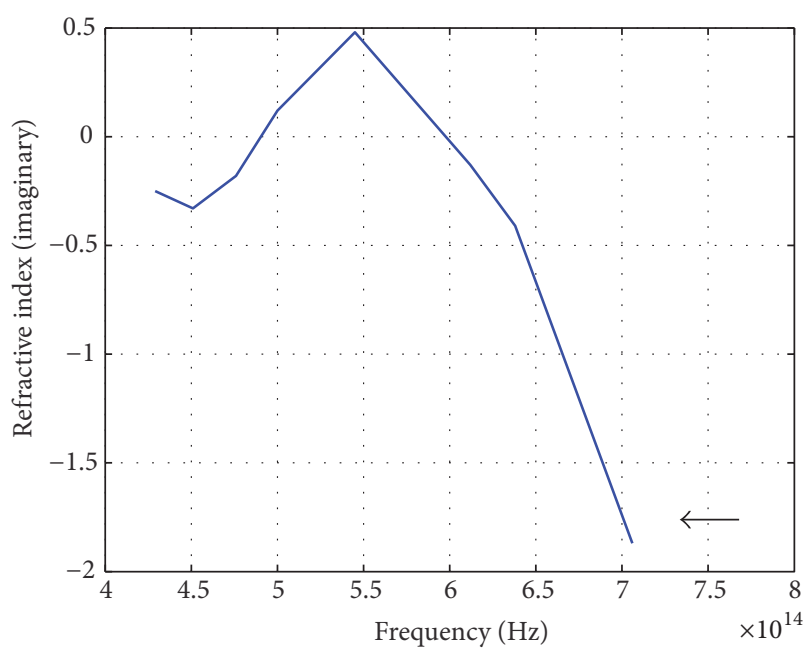

FIGURE 21: Plot showing how the refractive index (imaginary) changes with frequency for a radius ratio of 2 and fixed inner and outer radius value (arrow shows no further values can be achieved beyond a frequency of $7.1 \mathrm{THz}$ ).

\section{References}

[1] T. Hakkarainen, Electromagnetic nanophotonics: superlens imaging of dipolar emitters and cloaking in weak scattering [Ph.D. thesis], Department of Applied Physics, Aalto University, Espoo, Finland, 2012.

[2] E. Kallos, C. Argyropoulos, Y. Hao, and A. Alù, "Comparison of frequency responses of cloaking devices under nonmonochromatic illumination," Physical Review B, vol. 84, no. 4, Article ID 045102, 2011.

[3] W. Cai and V. Shalaev, Optical Metamaterials Fundamentals and Applications, Springer, New York, NY, USA, 2010.

[4] T. J. Cui, D. R. Smith, and R. Liu, Metamaterials Theory, Design, and Applications, Springer, New York, NY, USA, 2010.

[5] L. Raffensperger, “Ancient Romans' Color-Changing Goblet Was Feat of Nanotechnology," 2013, http://blogs.discovermagazine .com/d-brief/2013/08/29/ancient-romans-color-changing-goblet -was-feat-of-nanotechnology/\#.WGzjh0-LXnM.

[6] J. Pendry, Metamaterials and the Science of Invisibility, 2014, https://www.youtube.com/watch?v=5ZOV_9Jirp0.

[7] R. A. Shelby, D. R. Smith, and S. Schultz, "Experimental verification of a negative index of refraction," Science, vol. 292, no. 5514, pp. 77-79, 2001.

[8] Y. Hao and R. Mittra, FDTD Modeling of Metamaterials: Theory and Applications, Artech House, Norwood, Mass, USA, 1st edition, 2008.

[9] M. Sipos, Optics and cloaking in FDTD [B.Sc. thesis], Department of Physics, Ithaca College, 2008.

[10] C. Argyropoulos, E. Kallos, Y. Zhao, and Y. Hao, "Manipulating the loss in electromagnetic cloaks for perfect wave absorption," Optics Express, vol. 17, no. 10, pp. 8467-8475, 2009.

[11] C. Argyropoulos, E. Kallos, and Y. Hao, "Dispersive cylindrical cloaks under nonmonochromatic illumination," Physical Review E, vol. 81, no. 1, Article ID 016611, 2010.

[12] Y. Zhao, C. Argyropoulos, and Y. Hao, "Full-wave finitedifference time-domain simulation of electromagnetic cloaking structures," Optics Express, vol. 16, no. 9, pp. 6717-6730, 2008. 
[13] A. Dawood, "Finite difference time-domain modelling of metamaterials: GPU implementation of cylindrical cloak," Advanced Electromagnetics, vol. 2, no. 2, pp. 10-17, 2013.

[14] N. Anam and E. Zahir, "Analysis of FDTD cloaking in the visible frequency spectrum," in Proceedings of the 15th International Conference on Numerical Simulation of Optoelectronic Devices (NUSOD '15), pp. 65-66, IEEE, Taipei, Taiwan, September 2015.

[15] N. Anam and E. Zahir, "Courant stability number impact on high frequency EM cloaking using FDTD analysis," International Journal of Research in Computer Engineering \& Electronics (IJRCEE), vol. 3, no. 3, 2014.

[16] S. Zhang, W. Fan, K. J. Malloy, S. R. Brueck, N. C. Panoiu, and R. M. Osgood, "Demonstration of metal-dielectric negativeindex metamaterials with improved performance at optical frequencies," Journal of the Optical Society of America B, vol. 23, no. 3, pp. 434-438, 2006.

[17] N. Okada and J. B. Cole, "FDTD modeling of a cloak with a nondiagonal permittivity tensor," ISRN Optics, vol. 2012, Article ID 536209, 7 pages, 2012.

[18] J. A. Silva-Macědo, M. A. Romero, and B.-H. V. Borges, "An extended FDTD method for the analysis of electromagnetic field rotations and cloaking devices," Progress in Electromagnetics Research, vol. 87, pp. 183-196, 2008.

[19] A. A. Maradudin, Structured Surfaces as Optical Metamaterials, Cambridge University Press, 2011. 

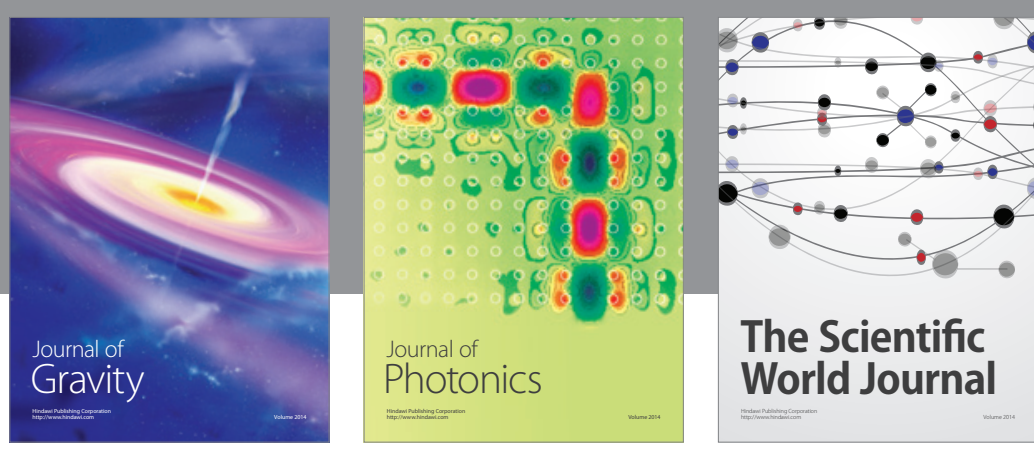

The Scientific World Journal
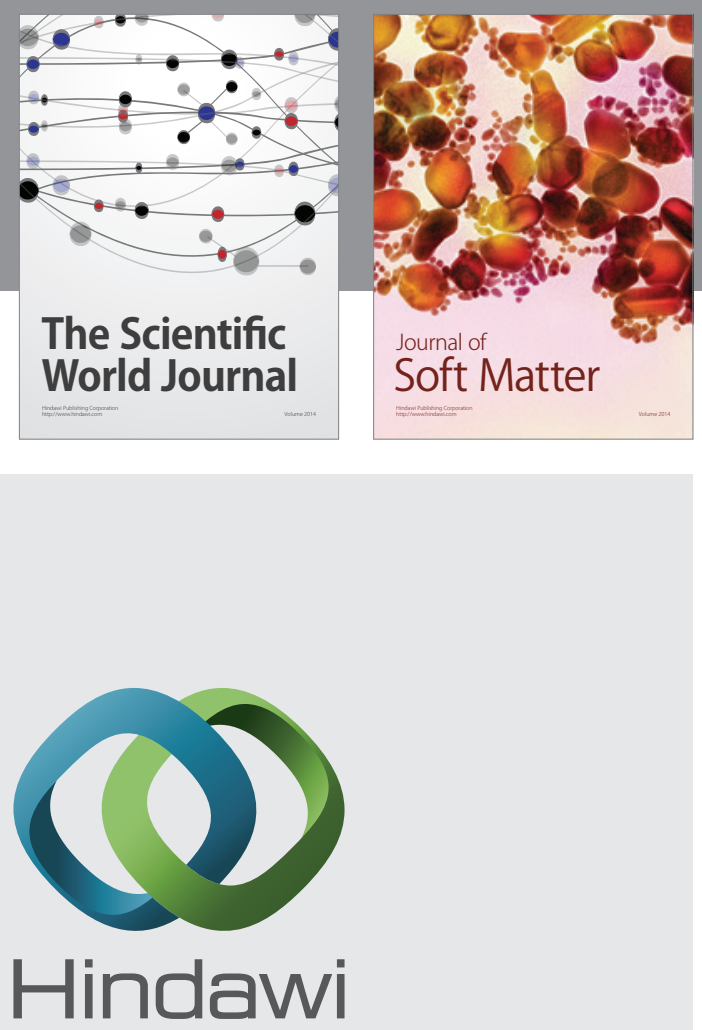

Submit your manuscripts at

https://www.hindawi.com
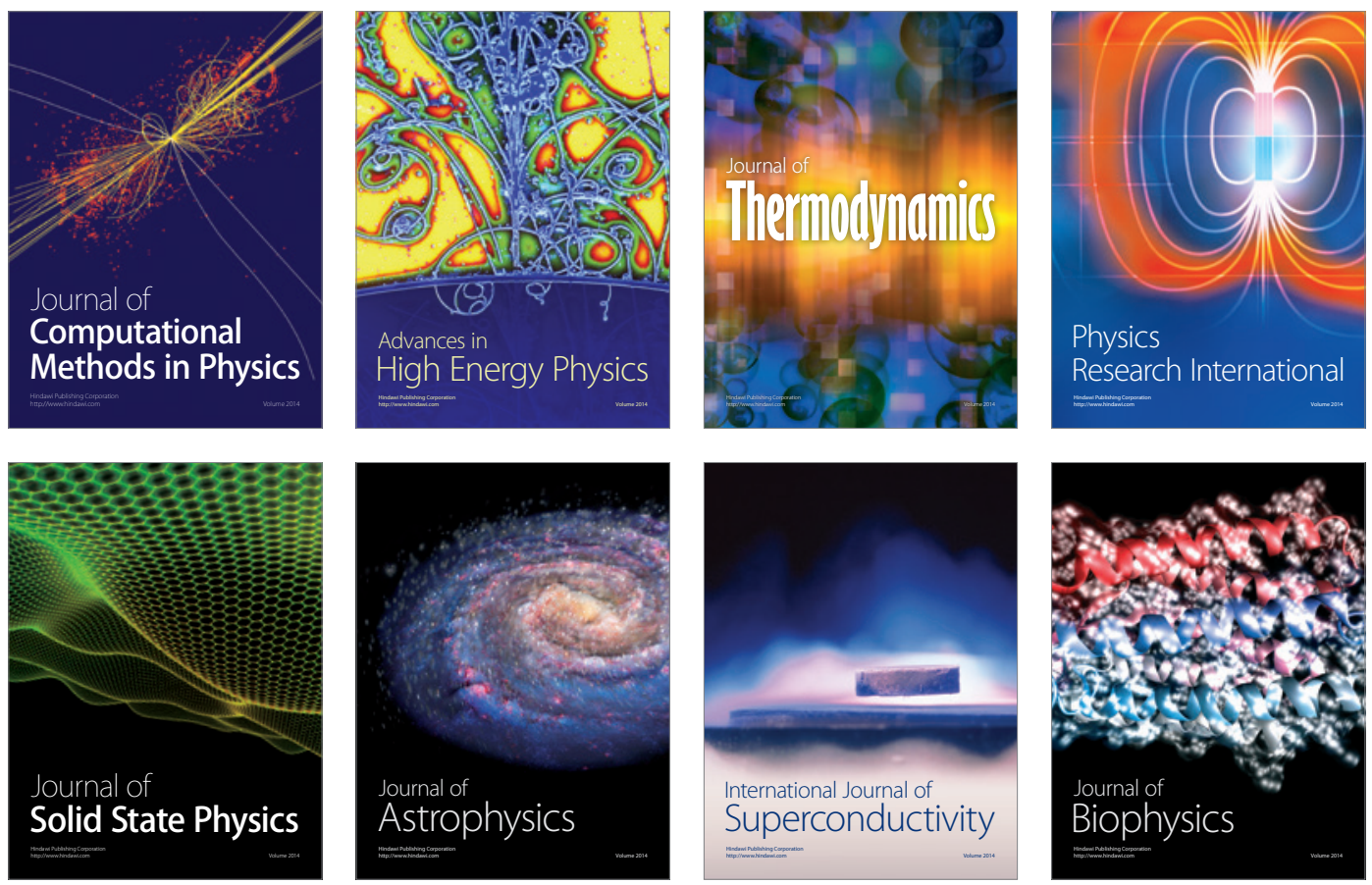
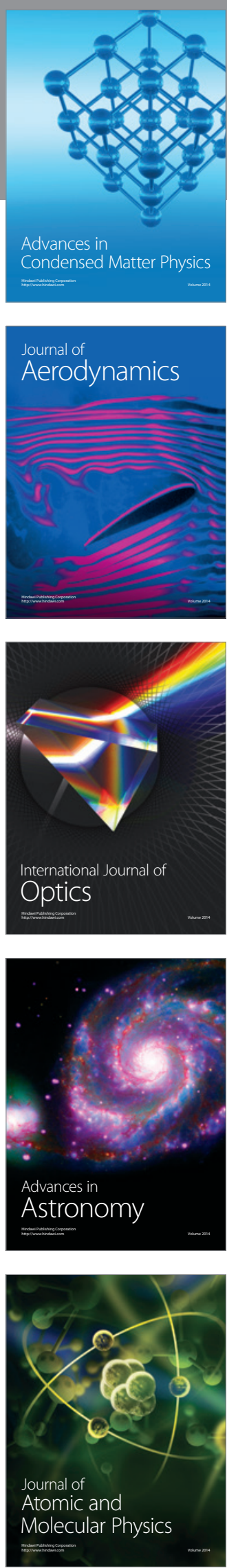